\title{
Lifetimes and Oscillator Strengths for Ultraviolet Transitions in Singly-Ionized Lead
}

\author{
N. Heidarian ${ }^{1}$, R. E. Irving ${ }^{1}$, A. M. Ritchey ${ }^{2}$, S. R. Federman ${ }^{1}$, D. G. Ellis ${ }^{1}$, S. Cheng ${ }^{1}$, \\ L. J. Curtis ${ }^{1}$, and W. A. Furman ${ }^{3}$
}

\begin{abstract}
We present the results of lifetime measurements made using beam-foil techniques on levels of astrophysical interest in $\mathrm{Pb}$ II producing lines at $1203.6 \AA$ $\left(6 s 6 p^{2}{ }^{2} D_{3 / 2}\right)$ and $1433.9 \AA\left(6 s^{2} 6 d^{2} D_{3 / 2}\right)$. We also report the first detection of the $\mathrm{Pb}$ II $\lambda 1203$ line in the interstellar medium (ISM) from an analysis of archival spectra acquired by the Space Telescope Imaging Spectrograph (STIS) onboard the Hubble Space Telescope (HST). The oscillator strengths derived from our experimental lifetimes for $\mathrm{Pb}$ II $\lambda \lambda 1203,1433$ are generally consistent with recent theoretical results, including our own relativistic calculations. Our analysis of high-resolution HST/STIS spectra helps to confirm the relative strengths of the $\mathrm{Pb}$ II $\lambda \lambda 1203,1433$ lines. However, the oscillator strength that we obtain for $\mathrm{Pb}$ II $\lambda 1433(0.321 \pm 0.034)$ is significantly smaller than earlier theoretical values, which have been used to study the abundance of $\mathrm{Pb}$ in the ISM. Our revised oscillator strength for $\lambda 1433$ yields an increase in the interstellar abundance of $\mathrm{Pb}$ of 0.43 dex over determinations based on the value given by Morton, indicating that the depletion of $\mathrm{Pb}$ onto interstellar dust grains is less severe than previously thought.
\end{abstract}

Subject headings: atomic data — ISM: abundances — ISM: atoms — methods: laboratory - ultraviolet: ISM

\section{INTRODUCTION}

Lead $(Z=82)$ is the heaviest element thus far detected in the interstellar medium (ISM), and is one of only a handful of elements heavier than zinc $(Z>30)$ that have been detected

\footnotetext{
${ }^{1}$ Department of Physics and Astronomy, University of Toledo, Toledo, OH 43606; negar.heidarianboroujeni@rockets.utoledo.edu, richard.irving@utoledo.edu, steven.federman@utoledo.edu, david.ellis@utoledo.edu, song.cheng@utoledo.edu, larry.curtis@utoledo.edu

${ }^{2}$ Department of Astronomy, University of Washington, Seattle, WA 98195; aritchey@astro.washington.edu

${ }^{3}$ Reed College, Portland, OR, 97202; afurman@reed.edu
} 
via ultraviolet interstellar absorption lines. Knowledge of the interstellar abundances of heavy elements yields insight into the processes of stellar nucleosynthesis responsible for the production of elements beyond the iron peak (i.e., the slow and rapid neutron-capture processes). Accurate gas-phase abundances for rare heavy elements can also provide us with a better understanding of the depletion processes by which atoms condense onto interstellar dust grains. With an ionization potential of $15.0 \mathrm{eV}, \mathrm{Pb}$ II is the dominant form of lead in the neutral ISM. Observations of the $6 s^{2} 6 p^{2} P_{1 / 2}^{\mathrm{o}}-6 s^{2} 6 d^{2} D_{3 / 2}$ transition of $\mathrm{Pb}$ II at $1433.906 \AA$ with the Goddard High-Resolution Spectrograph onboard the Hubble Space Telescope (HST) have been used to study the abundance of $\mathrm{Pb}$ in just two sight lines (Cardelli 1994; Welty et al. 1995). Additional detections of the $\mathrm{Pb}$ II $\lambda 1433$ line are available in archival Space Telescope Imaging Spectrograph (STIS) data (A. M. Ritchey et al., in preparation). However, the total number of $\mathrm{Pb}$ II detections in the ISM remains small, and, as a result, neither the abundance nor the depletion behavior of interstellar $\mathrm{Pb}$ are very well constrained.

In this paper, we report the first detection in the ISM of the $6 s^{2} 6 p^{2} P_{1 / 2}^{\mathrm{o}}-6 s 6 p^{2}{ }^{2} D_{3 / 2}$ transition of $\mathrm{Pb}$ II at $1203.616 \AA$. We discovered this line by co-adding high-resolution $H S T$ /STIS archival spectra for over 100 individual sight lines. A preliminary examination indicated that the $\mathrm{Pb}$ II $\lambda 1203$ line was stronger than $\lambda 1433$. However, an accurate oscillator strength $(f$-value) for the $\lambda 1203$ line was not available. In the compilation by Morton (2000), for example, there is no $f$-value listed for the $\mathrm{Pb}$ II transition at $1203.6 \AA$. Theoretical transition probabilities for $\mathrm{Pb}$ II $\lambda 1203$ and $\lambda 1433$ were reported by Colón \& Alonso-Medina (2001). However, these calculations, as well as those of Safronova et al. (2005), yield an $f$-value for the $\lambda 1433$ line that is significantly smaller than those from earlier theoretical efforts (Migdalek 1976; Cardelli et at. 1993), which were the basis for the $f$-value listed by Morton (2000) for $\mathrm{Pb}$ II $\lambda 1433$. Here, we present the first experimentally determined oscillator strengths for the Pb II transitions at 1203.6 and $1433.9 \AA$, obtained from lifetime measurements made using beam-foil techniques.

The remainder of this paper is organized as follows. In Section 2, we describe the analysis of archival $H S T$ /STIS spectra, which we use to derive empirically the relative $f$-values of the $\mathrm{Pb}$ II $\lambda 1203$ and $\lambda 1433$ lines. In Section 3, we discuss the lifetime measurements and the resulting oscillator strengths for several ultraviolet transitions in $\mathrm{Pb}$ II. In Section 4, we present our own theoretical calculations of the $\mathrm{Pb}$ II oscillator strengths, and compare these with our experimental results and with previous theoretical determinations. We summarize our conclusions in Section 5. 


\section{ASTRONOMICAL OBSERVATIONS}

In an effort to search for previously undetected interstellar absorption lines in the far ultraviolet, we co-added high-resolution HST/STIS archival spectra for large numbers of sight lines, producing high signal-to-noise ratio $(\mathrm{S} / \mathrm{N})$ composite spectra for different wavelength regions. Only spectra obtained with the E140H grating were considered. In addition, only sight lines that showed measurable interstellar absorption from the weak $\mathrm{O}$ I line at 1355.598 A were included. All spectra were aligned in velocity space, based on the position of the strongest O I $\lambda 1355$ absorption component, before co-adding, and were shifted to the laboratory rest frame. One absorption feature that was readily apparent in the composite spectra had a wavelength of $1203.616 \pm 0.003 \AA$, precisely equal to that expected for the $\mathrm{Pb}$ II line at $1203.616 \AA$. Figure 1 shows a portion of the co-added spectrum, obtained from observations of 104 individual sight lines, showing the feature, which is detected with a significance of $9 \sigma$. We checked for other possible identifications for this feature using compilations for atomic lines (Morton 2000, 2003) and molecular lines (e.g., Abgrall et al. 1993a, 1993b; Morton \& Noreau 1994), but could not find any other reasonable identification other than $\mathrm{Pb}$ II $\lambda 1203$.

Since the newly-discovered $\mathrm{Pb}$ II line did not have an accurate $f$-value (prior to this study), we sought to determine the $f$-value empirically by comparing the $\lambda 1203$ feature with $\mathrm{Pb}$ II $\lambda 1433$. Of the 104 sight lines originally used to detect the $\lambda 1203$ feature, only 34 also had high-resolution HST/STIS spectra covering the Pb II $\lambda 1433$ line. For these 34 sight lines, small spectral segments covering the 1203 and $1433 \AA$ regions were extracted from the STIS data, and were normalized by dividing each segment by its mean flux. The normalized spectra were then averaged, yielding a S/N of 100 at $1203 \AA$ and 240 at $1433 \AA$. This procedure ensures that the column density associated with the resulting $\mathrm{Pb}$ II absorption features corresponds to the average $\mathrm{Pb}$ II column density for this particular set of sight lines, assuming the $\mathrm{Pb}$ II lines are optically thin. While a more sophisticated weighting scheme could have been used to produce higher $\mathrm{S} / \mathrm{N}$ composite spectra, the column densities associated with the $\mathrm{Pb}$ II $\lambda 1203$ and $\lambda 1433$ features resulting from such a scheme would no longer be a simple average, and would not necessarily be identical. Table 1 lists the

background stars and the relevant information for the HST/STIS data sets used to produce the average spectra.

The $\mathrm{Pb}$ II $\lambda 1203$ and $\lambda 1433$ features appearing in the average spectra were fit using the profile synthesis code ISMOD (see Sheffer et al. 2008). For these syntheses, the $f$-value of the $\lambda 1433$ line was assumed to be $f_{1433}=0.321$, which is the value we obtain from our beamfoil experiments (see Section 3 ). The $f$-value of the $\lambda 1203$ line was varied until the column densities resulting from the two fits were identical for the same Doppler $b$-value. With this 
procedure, we find $f_{1203}=0.752$ for $N(\mathrm{~Pb} \mathrm{II})=1.18 \times 10^{11} \mathrm{~cm}^{-2}$ and $b=2.6 \mathrm{~km} \mathrm{~s}^{-1}$. The fitted equivalent widths for the two lines are $W_{1203}=1.11 \pm 0.23 \mathrm{~m} \AA$ and $W_{1433}=$ $0.68 \pm 0.11 \mathrm{~m} \AA$, while the fitted wavelengths are $1203.616 \pm 0.003 \AA$ and $1433.903 \pm 0.003 \AA$. The profile fits themselves are shown in Figure 2. From these results, we find that the ratio of $f$-values is $f_{1203} / f_{1433}=2.34 \pm 0.43$, regardless of the value assumed for $f_{1433}$. If the $\mathrm{Pb}$ II lines are indeed optically thin, then the $f$-value ratio should be given by $f_{1203} / f_{1433}=$ $\left(\lambda_{1433} / \lambda_{1203}\right)^{2} W_{1203} / W_{1433}$. Use of this equation with the above equivalent widths yields an $f$-value ratio of 2.32 , in agreement with the profile synthesis results.

\section{EXPERIMENTAL DETAILS}

\subsection{Measurements}

Lifetimes were measured using beam foil techniques at the Toledo Heavy Ion Accelerator (THIA; Haar et al. 1993; Schectman et al. 2000). $\mathrm{Pb}^{+}$ions were produced by a Danfysik Model 911A Universal Ion Source, extracted at $20 \mathrm{kV}$, magnetically analyzed, and then accelerated to a total kinetic energy of either $205 \mathrm{keV}$ or $180 \mathrm{keV}$. The two different beam energies were used to study possible systematic effects (see, e.g., Federman et al. 1992). Using an electrostatic switchyard, magnetically selected ions were steered toward carbon foils with thicknesses ranging from 2.2 to $2.4 \mu \mathrm{g} \mathrm{cm}^{-2}$. Typical beam currents were $\sim 100 \mathrm{nA}$ to minimize foil breakage. Emission lines were analyzed with an Acton $1 \mathrm{~m}$ normal incidence vacuum ultraviolet monochromator. Post foil velocities were estimated to range from 0.391 to $0.392 \mathrm{~mm} \mathrm{~ns}^{-1}$ for the $205 \mathrm{keV}$ beam and from 0.419 to $0.420 \mathrm{~mm} \mathrm{~ns}^{-1}$ for the $180 \mathrm{keV}$ beam. Uncertainties in foil thickness and in the energy calibration of the accelerator will impact the precision of these post foil velocity determinations. We measured decay curves for the $6 s^{2} 6 d^{2} D_{3 / 2}$ level at $69,740 \mathrm{~cm}^{-1}$ and the $6 s 6 p^{2}{ }^{2} D_{3 / 2}$ level at $83,083 \mathrm{~cm}^{-1}$ using the resonance lines at 1433.9 and $1203.6 \AA$. Decay curves were also measured for the $6 s 6 p^{2}$ ${ }^{2} D_{5 / 2}$ level using the transition at $1335.20 \AA$, and the $6 s^{2} 6 d^{2} D_{5 / 2}$ level was measured from the transition at $1822.05 \AA$. All decay curves and spectra were normalized by means of an optical monitor.

Lifetimes were obtained through analysis of the decay curves. Two exponentials were required for the $6 s^{2} 6 d^{2} D_{3 / 2}$ level, since there is a repopulation coming from the cascade of the $5 f^{2} F_{5 / 2}$ level to the level of interest; we therefore employed the method of Arbitrarily Normalized Decay Curves (ANDC; Curtis et al. 1971) in the analysis, which enabled us to extract the lifetime of the primary decay for $6 s^{2} 6 d^{2} D_{3 / 2}$. Theoretical calculations indicate a lifetime for this cascade of 3.61 ns (Colón \& Alonso-Medina 2001) and 5.55 ns (AlonsoMedina 1996) and the latter one is consistent with other experimental values (Gorshkov \& 
Verolainen 1985; Alonso-Medina 1997) as well as our ANDC analysis. For the $6 s 6 p^{2}{ }^{2} D_{3 / 2}$ level, we did not find a noticeable cascade; the ANDC analysis gave the same primary lifetime as the one we obtained from a single-exponential fit. The lifetime for the $6 s 6 p^{2}{ }^{2} D_{5 / 2}$ level was obtained by means of a single-exponential fit as well. Measuring the decay curve for this level (using the $\lambda 1335.2$ line) was challenging since there are two possibly strong potential blends coming from resonance lines of C II at 1334.5 and $1335.7 \AA$. The $\mathrm{C}$ II $\lambda 1335.7$ line is twice as strong as $\lambda 1334.5$ (Morton 2003). Thus, to avoid the possible blending from C II $\lambda 1335.7$, we took the measurements at $1334.9 \AA$. The lifetime for the $6 s^{2} 6 d{ }^{2} D_{5 / 2}$ level was also obtained from a single-exponential fit using the $\lambda 1822$ line. No evidence was found for a potential cascade in this line either.

Finally, in order to derive oscillator strengths from lifetimes, we need the branching fractions for the transitions we have measured. We adopted the values from Colón \& AlonsoMedina (2001) because the various branches span too large a range for our experimental techniques. For $\lambda 1433$, the branching fraction from Colón \& Alonso-Medina (2001) is 0.797, while, for $\lambda 1203$, it is essentially 1.000. For comparison, the values from our own theoretical calculations, described in Section 4, are 0.770 and 0.999 for $\lambda 1433$ and $\lambda 1203$, respectively. For $\lambda 1335$ and $\lambda 1822$, there are no significant additional decay channels.

\subsection{Results}

Decay curves are shown in Figures 3, 4, 5 and 6 for $\lambda 1433, \lambda 1203, \lambda 1335$ and $\lambda 1822$ respectively. Reported lifetimes correspond to the weighted averages of the lifetimes obtained with the two beam energies. Uncertainties are derived from the weighted uncertainty of exponential fit errors and systematic errors (estimated from the range in lifetimes measured from the two different energies), added in quadrature.

The results for lifetime measurements are shown in Table 2 and the resulting oscillator strengths are given in Table 3 . The values for $\lambda 1796$ and $\lambda 1449$ are derived from the measured lifetimes and the corresponding branching fractions from Colón \& Alonso-Medina (2001). Previous theoretical lifetimes and oscillator strengths are also reported in Tables 2 and 3. Our experimental oscillator strengths for $\mathrm{Pb}$ II $\lambda \lambda 1203,1433$ yield an $f$-value ratio of $f_{1203} / f_{1433}=2.3 \pm 0.2$, which is in very good agreement with the ratio we obtain from our analysis of high-resolution HST/STIS spectra (see Section 2).

As mentioned above, the decay of $\lambda 1433$ is significantly affected by a cascade, whereas

the $\lambda 1203$ decay may be treated as essentially cascade-free. Our theoretical calculations, discussed in the next section, help to explain this; the $6 s^{2} 5 f^{2} F_{5 / 2}^{\circ}$ level decays to $6 s^{2} 6 d$ 
${ }^{2} D_{3 / 2}$ by means of a strong one-electron transition with a transition probability of $A \approx 0.55$ $\mathrm{ns}^{-1}$, whereas its decay to the $6 s 6 p^{2}{ }^{2} D_{3 / 2}$ level is nominally a two-electron change, and thus relies on configuration mixing resulting in a smaller value of $A \approx 0.025 \mathrm{~ns}^{-1}$. This helps to confirm our ability to detect the presence or absence of significant cascade effects in our measurements.

\section{THEORETICAL CALCULATIONS}

The ion $\mathrm{Pb}^{+}$has 81 electrons; its ground electronic configuration is [Xe $] 4 f^{14} 5 d^{10} 6 s^{2} 6 p$, where $[\mathrm{Xe}]$ denotes the xenon-like core of 54 electrons (Earls \& Sawyer 1935). The ground state is designated as $6 s^{2} 6 p^{2} P_{1 / 2}^{\mathrm{o}}$ in Russell-Saunders (LS) coupling notation. The lowest even-parity configurations are $6 s 6 p^{2}, 6 s^{2} 7 s$ and $6 s^{2} 6 d$. The $6 s 6 p^{2}$ configuration gives rise to four LS-coupled terms: ${ }^{4} P,{ }^{2} D,{ }^{2} P$, and ${ }^{2} S$. We have calculated the transition rates from the ground $6 p$ to all these excited configurations, though we are especially interested in the resonance lines involving the lowest ${ }^{2} D_{3 / 2}$ levels: $6 s^{2} 6 p^{2} P_{1 / 2}^{\mathrm{o}} \longrightarrow 6 s^{2} 6 d^{2} D_{3 / 2} \quad(\lambda 1433)$ and $6 s^{2} 6 p^{2} P_{1 / 2}^{\mathrm{o}} \longrightarrow 6 s 6 p^{2}{ }^{2} D_{3 / 2} \quad(\lambda 1203)$.

Several calculations have been made recently with varying results. The one most relevant for us here is that of Colón \& Alonso-Medina (2001), who point out that the ${ }^{2} D$ terms are subject to considerable interaction between the $6 s 6 p^{2}$ and $6 s^{2} 6 d$ configurations. Their calculation adopted the method of Cowan (1981) in which relativistic Hartree-Fock (HFR) wavefunctions, configuration interactions in intermediate coupling, and a fit to observed energy levels are used to determine the mixing coefficients and transition probabilities. In another recent effort, Safronova et al. (2005) presented a calculation of the $6 s^{2} n d$ series, using relativistic many-body perturbation theory. Earlier theoretical efforts (Migdalek 1976; Cardelli et at. 1993), though consistent among themselves, produced $f$-values that are significantly larger than those from the two recent calculations for the $6 s^{2} 6 d$ transitions.

We have done a multi-configuration Dirac Hartree Fock (MCDHF) calculation using GRASP2K (Jönsson et al. 2007; Jönsson et al. 2013), which is a general atomic structure program that includes both electron correlation and special relativity. Self-consistent multiconfiguration equations using the Dirac-Coulomb Hamiltonian are solved to give the atomic state functions as linear combinations of relativistic configuration state functions (RCSFs) based on Dirac orbitals. Relativistic corrections, including the Breit interaction, are then added to the Hamiltonian and a relativistic configuration-interaction (RCI) calculation is performed in which the theoretical energy levels are produced as the eigenvalues of the resulting energy matrix. 
We treat the $\mathrm{Pb}$ II system as a core of 78 electrons with an additional 3 valence electrons in the $n=6$ shell. We begin with a reference solution using the three non-relativistic configurations $6 s^{2} 6 p, 6 s 6 p^{2}$, and $6 s^{2} 7 s$, which contain the lowest energy levels. This involves 25 Dirac orbitals, all of which are varied to obtain a fully self-consistent solution. We then produce solutions of increasing complexity in two ways. (1) We add virtual orbitals of successively higher values of the $n$ and $l$ quantum numbers, with RCSFs formed by single and double electron substitutions from the reference solution. (2) We take account of corevalence correlation in a limited way by allowing single substitutions from the $5 d$ orbital. In this way, we develop a basic solution with 32 Dirac orbitals, namely all those with $n+l \leq 8$, and 5,590 RSCFs, most of which $(5,173)$ involve the $5 d^{9}$ group. This solution is optimized on the 15 lowest levels (11 even, 4 odd), and already shows significant interaction between the two ${ }^{2} D_{3 / 2}$ levels.

Next, we add three layers of virtual orbitals, the outer layer being $6 h, 7 g, 8 f, 9 d$, $10 p, 11 s$. At each stage, we use single and double electron replacements, including the $5 d^{9}$ subshell, and vary only the outer layer, keeping the other orbitals fixed. We now compute the even-parity levels separately from the odd. We finish with a pair of solutions having 61 relativistic orbitals, namely all those with $n+l \leq 11$. For the even-parity solution, we have 114,966 RCSFs, while, for the odd, we have 21,777. For the even solution, the radial functions for the outer orbitals are optimized on the lowest 9 even-parity levels, including the two ${ }^{2} D$ terms, with standard $(2 j+1)$ weighting. For the odd solution, we optimize on the 4 lowest levels, namely the $6 p$ and $7 p$ doublets. After obtaining these MCDHF solutions, we continue with the GRASP2K program suite, to do RCI calculations, including the Breit interaction, again one for each parity, repeating for each step as virtual orbitals are added. From these calculations, we get the lowest 13 energy eigenvalues. We then compute the oscillator strengths for the dipole transitions between these levels. Note that the relativistic treatment automatically includes spin-changing transitions (i.e., intercombination lines).

Our theoretical results are shown in Tables 2 and 3, along with our experimental results and previously published results. The estimated precision for the theoretical $f$-values takes into account both the change in computed values as additional orbitals are added to the calculation, and the difference between the Babushkin (length) and the Coulomb (velocity) gauges. The calculated oscillator strengths have been corrected for the differences between observed and calculated transition energies. These energy differences are at most about $1 \%$. The calculated ratio of oscillator strengths is $f_{1203} / f_{1433}=4.20 \pm 0.23$. 


\section{SUMMARY AND CONCLUSIONS}

We have discussed various determinations of the oscillator strengths for ultraviolet transitions in $\mathrm{Pb}$ II. From an analysis of high-resolution HST/STIS archival spectra, we find that the ratio of the $f$-values of the $\mathrm{Pb}$ II $\lambda 1203$ and $\lambda 1433$ lines is $f_{1203} / f_{1433}=2.34 \pm 0.43$. Our detection of the $\mathrm{Pb}$ II $\lambda 1203$ feature in composite HST/STIS spectra represents the first detection of this line in the ISM. Lifetimes were measured using beam-foil techniques for several levels in $\mathrm{Pb}$ II. The resulting oscillator strengths for the $\mathrm{Pb}$ II $\lambda 1203$ and $\lambda 1433$ transitions are consistent with recent theoretical results, including our own relativistic calculations. Moreover, the $f$-value ratio from our beam-foil experiments $\left(f_{1203} / f_{1433}=2.3 \pm 0.2\right)$ is in very good agreement with our result from astronomical spectra. However, the $f$-value we obtain for $\mathrm{Pb}$ II $\lambda 1433(0.321 \pm 0.034)$ is significantly smaller than those from earlier theoretical calculations, which were the basis for the $f$-value listed by Morton (2000) for this transition. This is not surprising considering the complexities of this three-valenceelectron system: core-valence correlation, relativistic effects including the Breit interaction, and strong configuration mixing. Our GRASP2K calculation, which does address these com-

plexities and is most consistent with our THIA results as shown in Table 3, is probably the best theoretical treatment of these transitions to date. The earlier calculations were based on one-active-electron approximations which did not adequately account for these complexities.

Our revised $f$-value for $\mathrm{Pb}$ II $\lambda 1433$ yields an increase in the gas-phase interstellar abundance of $\mathrm{Pb}$ of 0.43 dex over determinations based on the Morton (2000) value. With this revision, it appears that the depletion of $\mathrm{Pb}$ onto interstellar dust grains is not as severe as previously thought. However, additional detections of Pb II in the ISM will ultimately be needed to fully understand the depletion behavior of this rare heavy element.

This work was supported by grant HST-AR-12123.001-A from the Space Telescope Science Institute. W. A. F. participated in the Research Experiences for Undergraduates (REU) Program of the National Science Foundation under award number 1262810. A. M. R. acknowledges support from the Kenilworth Fund of the New York Community Trust.

\section{REFERENCES}

Abgrall, H., Roueff, E., Launay, F., Roncin, J. Y., \& Subtil, J. L. 1993a, A\&AS, 101, 273

Abgrall, H., Roueff, E., Launay, F., Roncin, J. Y., \& Subtil, J. L. 1993b, A\&AS, 101, 323

Alonso-Medina, A. 1996, JQSRT, 55, 151 
Alonso-Medina, A. 1997, PhyS, 55, 49

Cardelli, J. A., Federman, S. R., Lambert, D. L., \& Theodosiou, C. E. 1993, ApJL, 416, L41

Cardelli, J. A. 1994, Sci, 265, 209

Colón, C., \& Alonso-Medina, A. 2001, CaJPh, 79, 999

Cowan, R. D. 1981, The Theory of Atomic Structure and Spectra (Berkeley: University of California Press)

Curtis, L. J., Berry, H. G., \& Bromander, J. 1971, PhLA, 34, 169

Earls, L. T., \& Sawyer, R. A. 1935, PhRv, 47, 115

Federman, S. R., Beideck, D. J., Schectman, R. M., \& York, D. G. 1992, ApJ, 401, 367

Gorshkov, V. N., \& Verolainen, Y. F. 1985, OptSp, 58, 848

Haar, R. R., Beideck, D. J., Curtis, L. J., et al. 1993, NIMPB, 79, 746

Jönsson, P., He, X., Froese Fischer, C., \& Grant, I. P. 2007, The GRASP2K Relativistic Atomic Structure Package, Computer Physics Communications, 177, 597

Jönsson, P., Gaigalas, G., Bieron, C., Froese Fischer, C., \& Grant, I. P. 2013, New Version: GRASP2K Relativistic Atomic Structure Package, Computer Physics Communications, 184, 2197

Migdalek, J. 1976, JQSRT, 16, 265

Morton, D. C. 2000, ApJS, 130, 403

Morton, D. C. 2003, ApJS, 149, 205

Morton, D. C., \& Noreau, L. 1994, ApJS, 95, 301

Safronova, U. I., Safronova, M. S., \& Johnson, W. R. 2005, PhRvA, 71, 052506

Schectman, R. M., Cheng, S., Curtis, L. J., et al. 2000, ApJ, 542, 400

Sheffer, Y., Rogers, M., Federman, S. R., et al. 2008, ApJ, 687, 1075

Welty, D. E., Hobbs, L. M., Lauroesch, J. T., Morton, D. C., \& York, D. G. 1995, ApJL, 449, L135

This preprint was prepared with the AAS IATEX macros v5.2. 
Table 1. HST/STIS Data Sets

\begin{tabular}{|c|c|c|c|c|c|c|c|}
\hline Star & Data Set & $\begin{array}{l}\text { Exp. Time } \\
\text { (s) }\end{array}$ & $\begin{array}{c}\text { Cen. Wave } \\
(\AA)\end{array}$ & Star & Data Set & $\begin{array}{l}\text { Exp. Time } \\
\text { (s) }\end{array}$ & $\begin{array}{c}\text { Cen. Wave } \\
(\AA)\end{array}$ \\
\hline \multirow[t]{2}{*}{ HD 15137} & o5lh02 & 2957 & 1271 & HD 121968 & o57r02 & 4457 & 1271 \\
\hline & o6lz06 & 1200 & 1489 & & o57r02 & 8383 & 1453 \\
\hline \multirow[t]{2}{*}{ HD 23478} & o6lj01 & 2945 & 1271 & HD 122879 & ob2611 & 1990 & 1234 \\
\hline & o6lj01 & 1448 & 1453 & & o5c037 & 360 & 1271 \\
\hline \multirow[t]{3}{*}{ HD 24190} & ob2604 & 1750 & 1234 & & o5lh07 & 1416 & 1271 \\
\hline & o6lj02 & 2940 & 1271 & & obkr37 & 600 & 1271 \\
\hline & o6lj02 & 1448 & 1453 & & o6lz57 & 600 & 1489 \\
\hline \multirow[t]{2}{*}{ HD 62542} & obik01 & 13298 & 1307 & HD 124314 & o54307 & 1466 & 1271 \\
\hline & obik02 & 13298 & 1343 & & o6lz58 & 300 & 1489 \\
\hline \multirow[t]{2}{*}{ HD 90087} & obie15 & 2038 & 1271 & HD 137595 & o6lj03 & 840 & 1271 \\
\hline & o6lz32 & 1200 & 1489 & & o6lj03 & 711 & 1453 \\
\hline \multirow[t]{2}{*}{ HD 93205} & o4qx01 & 1200 & 1234 & HD 147683 & o6lj06 & 2940 & 1271 \\
\hline & o4qx01 & 780 & 1416 & & o6lj06 & 1903 & 1453 \\
\hline \multirow[t]{2}{*}{ HD 93222} & o4qx02 & 1680 & 1234 & HD 148937 & obkr42 & 600 & 1271 \\
\hline & $\mathrm{o} 4 \mathrm{qx} 02$ & 1140 & 1416 & & o6f301 & 883 & 1380 \\
\hline \multirow[t]{2}{*}{ HD 93843} & o5lh04 & 1396 & 1271 & HD 177989 & o57r03 & 4557 & 1271 \\
\hline & o6lz40 & 300 & 1489 & & o57r03 & 8691 & 1453 \\
\hline \multirow[t]{2}{*}{ HD 99857} & o54301 & 3921 & 1271 & & o57r04 & 10356 & 1489 \\
\hline & o6lz44 & 1200 & 1489 & HD 195965 & o6bg01 & 415 & 1307 \\
\hline \multirow[t]{3}{*}{ HD 99872} & ob2603 & 2130 & 1234 & & o6bg01 & 300 & 1489 \\
\hline & o6lj0i & 1890 & 1271 & HD 201345 & ob2613 & 1746 & 1234 \\
\hline & o6lj0i & 1260 & 1453 & & o5c050 & 360 & 1271 \\
\hline \multirow[t]{3}{*}{ HD 99890} & obkj03 & 16857 & 1234 & & obkr50 & 600 & 1271 \\
\hline & obkr3k & 600 & 1271 & & o6359p & 360 & 1489 \\
\hline & obkj02 & 11990 & 1416 & HD 202347 & o5g301 & 830 & 1271 \\
\hline \multirow[t]{2}{*}{ HD 102065} & o4o001 & 7679 & 1234 & & o5g301 & 620 & 1453 \\
\hline & o4o001 & 1200 & 1416 & & o5g301 & 900 & 1489 \\
\hline \multirow[t]{2}{*}{ HD 103779} & o54302 & 1466 & 1271 & HD 209339 & o5lh0b & 1416 & 1271 \\
\hline & o63563 & 720 & 1489 & & o6lz92 & 1200 & 1489 \\
\hline \multirow[t]{3}{*}{ HD 104705} & o57r01 & 2400 & 1271 & HD 218915 & o57r05 & 2018 & 1271 \\
\hline & o57r01 & 3320 & 1453 & & o57r05 & 1300 & 1453 \\
\hline & o57r01 & 2900 & 1489 & & o57r05 & 1262 & 1489 \\
\hline \multirow[t]{3}{*}{ HD 108639} & ob2601 & 3730 & 1234 & HD 224151 & o54308 & 1496 & 1271 \\
\hline & o6lj0a & 1860 & 1271 & & o6lz96 & 300 & 1489 \\
\hline & o6lj0a & 1800 & 1453 & HD 232522 & obkj06 & 17166 & 1234 \\
\hline \multirow[t]{2}{*}{ HD 110434} & o6lj0b & 2130 & 1271 & & o5c08j & 1440 & 1271 \\
\hline & o6lj0b & 1020 & 1453 & & obkj05 & 12776 & 1416 \\
\hline \multirow[t]{2}{*}{ HD 114886} & o6lj0d & 1560 & 1271 & HD 303308 & o4qx04 & 2220 & 1271 \\
\hline & o6lj0d & 1590 & 1453 & & o4qx04 & 1560 & 1416 \\
\hline \multirow[t]{4}{*}{ HD 116852} & o5c01c & 360 & 1271 & CPD-59 2603 & o4qx03 & 5160 & 1234 \\
\hline & o8na03 & 5722 & 1271 & & o4qx03 & 3180 & 1416 \\
\hline & obkr1c & 600 & 1271 & & & & \\
\hline & o63571 & 720 & 1489 & & & & \\
\hline
\end{tabular}


Table 2. Lifetimes for the $6 s^{2} 6 d$ and $6 s 6 p^{2}$ Levels

\begin{tabular}{ccccc}
\hline \hline \multirow{2}{*}{ Level } & \multicolumn{4}{c}{$\tau(\mathrm{ns})$} \\
\cline { 2 - 5 } & THIA $^{\mathrm{a}}$ & GRASP2K & Other Theory & Ref. \\
\hline \multirow{2}{*}{$6 d^{2} D_{3 / 2}$} & $1.53(16)^{\mathrm{b}}$ & $1.772(32)$ & $1.32,1.16$ & 1,2 \\
$6 d^{2} D_{5 / 2}$ & $4.13(32)$ & $6.084(358)$ & $13.75,1.42$ & 1,2 \\
$6 s 6 p^{2}{ }^{2} D_{3 / 2}$ & $0.58(2)$ & $0.386(20)$ & 0.215 & 1 \\
$6 s 6 p^{2}{ }^{2} D_{5 / 2}$ & $1.97(14)$ & $0.700(28)$ & 0.447 & 1 \\
\hline
\end{tabular}

${ }^{a}$ Beam-foil results from the Toledo Heavy Ion Accelerator.

${ }^{b}$ Numbers in parentheses are uncertainties, shown in the last digits.

References. - (1) Colón \& Alonso-Medina 2001 - relativistic Hartree Fock (from quoted transition probability); (2) Safronova et al. 2005 - all-order Dirac Fock.

Table 3. Oscillator Strengths for ${ }^{2} P^{\mathrm{o}}-^{2} D$ Transitions

\begin{tabular}{cccccc}
\hline \hline \multirow{2}{*}{ Transition } & \multirow{2}{*}{ Wavelength } & \multicolumn{4}{c}{$f$-value } \\
\cline { 3 - 6 } & $(\AA)$ & THIA $^{\mathrm{a}}$ & GRASP2K & Other Theory & Ref. \\
\hline $6 p^{2} P_{1 / 2}^{\mathrm{o}}-6 d^{2} D_{3 / 2}$ & 1433.9 & $0.321(34)^{\mathrm{b}}$ & $0.268(5)$ & $0.869,0.86,0.372,0.4518$ & $1,2,3,4$ \\
$6 p^{2} P_{3 / 2}^{\mathrm{o}}-6 d^{2} D_{3 / 2}$ & 1796.67 & $0.064(7)$ & $0.063(1)$ & $0.100,0.074,0.06255$ & $1,3,4$ \\
$6 p^{2} P_{3 / 2}^{\mathrm{o}}-6 d^{2} D_{5 / 2}$ & 1822.05 & $0.179(14)$ & $0.123(7)$ & $0.880,0.054,0.5024$ & $1,3,4$ \\
$6 p^{2} P_{1 / 2}-6 s 6 p^{2}{ }^{2} D_{3 / 2}$ & 1203.62 & $0.75(3)$ & $1.124(58)$ & & 2.02 \\
$6 p^{2} P_{3 / 2}-6 s 6 p^{2}{ }^{2} D_{3 / 2}$ & 1449.35 & $0.0001(0)$ & $0.0011(3)$ & 0.0003 & 3 \\
$6 p^{2} P_{3 / 2}-6 s 6 p^{2}{ }^{2} D_{5 / 2}$ & 1335.20 & $0.204(14)$ & $0.573(23)$ & 0.897 & 3 \\
\hline
\end{tabular}

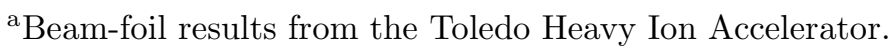

${ }^{b}$ Numbers in parentheses are uncertainties, shown in the last digits.

References. - (1) Migdałek 1976 - relativistic; (2) Cardelli et al. 1993 - Coulomb approximation with core polarization; (3) Colón \& Alonso-Medina 2001 - relativistic Hartree Fock (from quoted transition probability); (4) Safronova et al. 2005 - all-order Dirac Fock. 


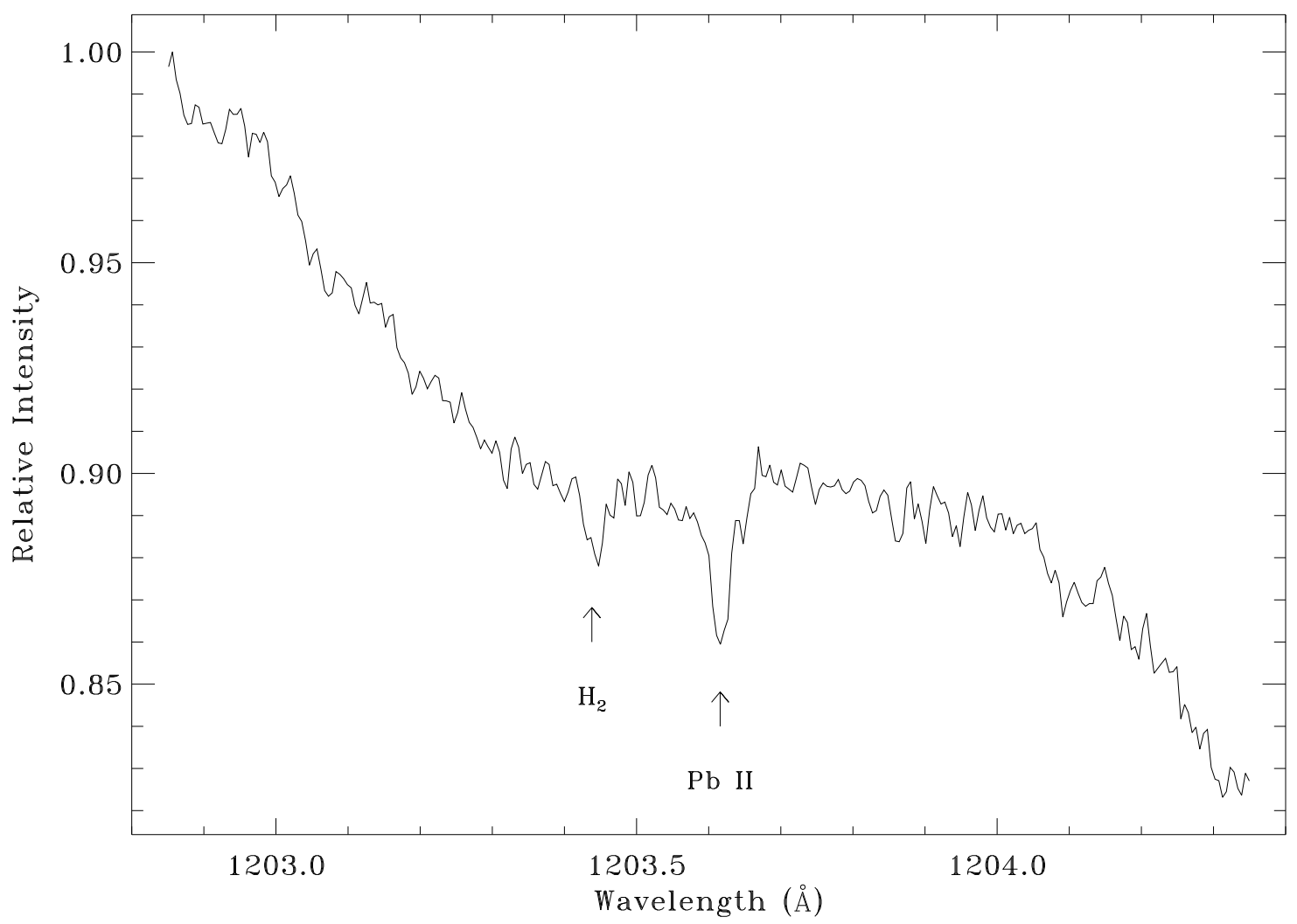

Fig. 1. - Composite high-resolution HST/STIS spectrum, obtained by co-adding data for 104 individual sight lines, covering the region surrounding the Pb II line at $1203.616 \AA$. The $\mathrm{Pb}$ II feature is detected at the $9 \sigma$ level in this co-added spectrum. The other detected feature near $1203.44 \AA$ is a line of vibrationally-excited $\mathrm{H}_{2}$, which is strong in some sight lines. 

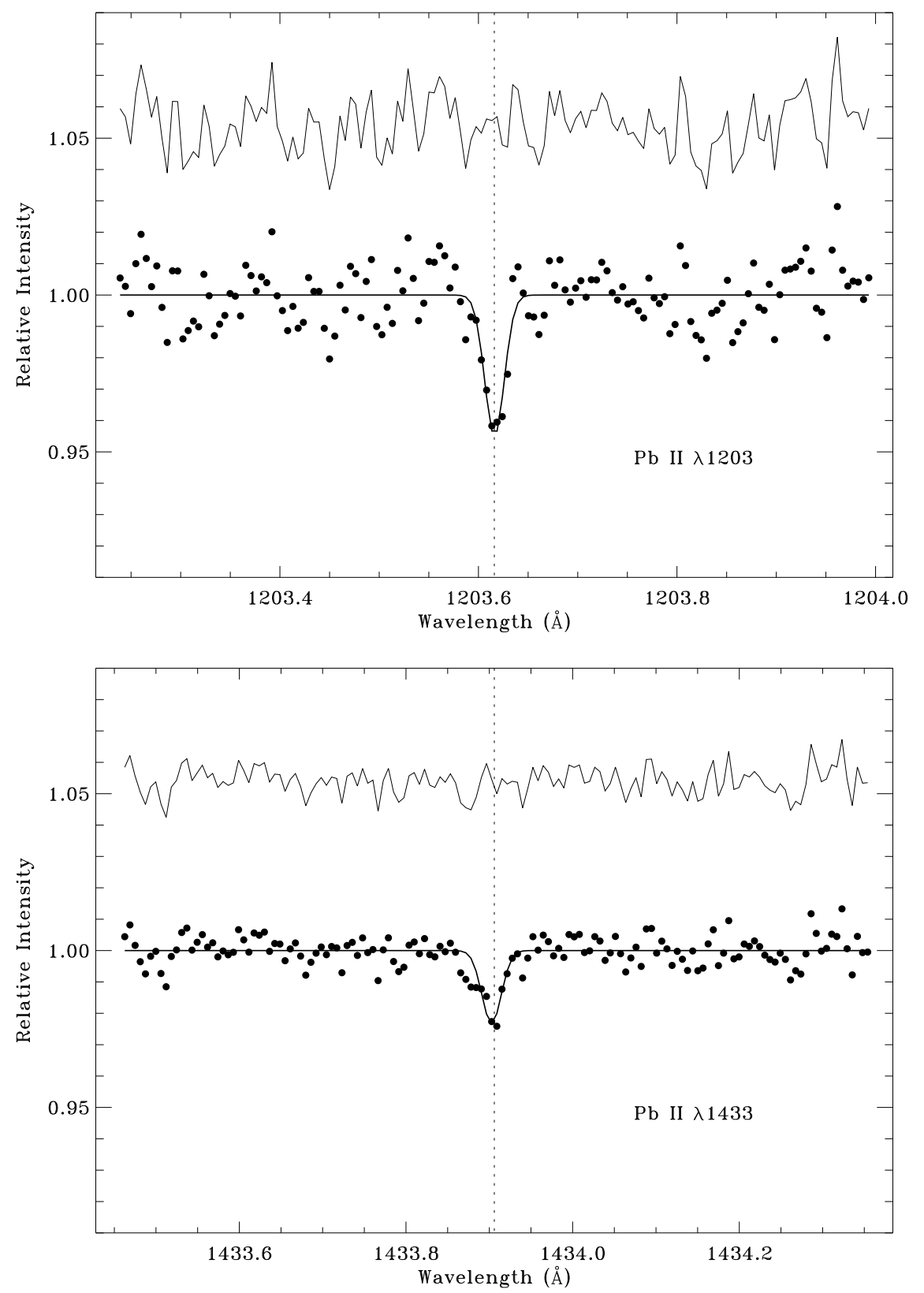

Fig. 2.- Profile synthesis fits to the Pb II $\lambda 1203$ and $\lambda 1433$ features appearing in the average spectra for 34 sight lines with high-resolution HST/STIS data covering both wavelength regions. Synthetic profiles are shown as solid lines passing through data points that represent the observed spectra. Residuals are plotted above each fit. The vertical dotted lines mark the expected positions of the $\mathrm{Pb}$ II absorption features. 


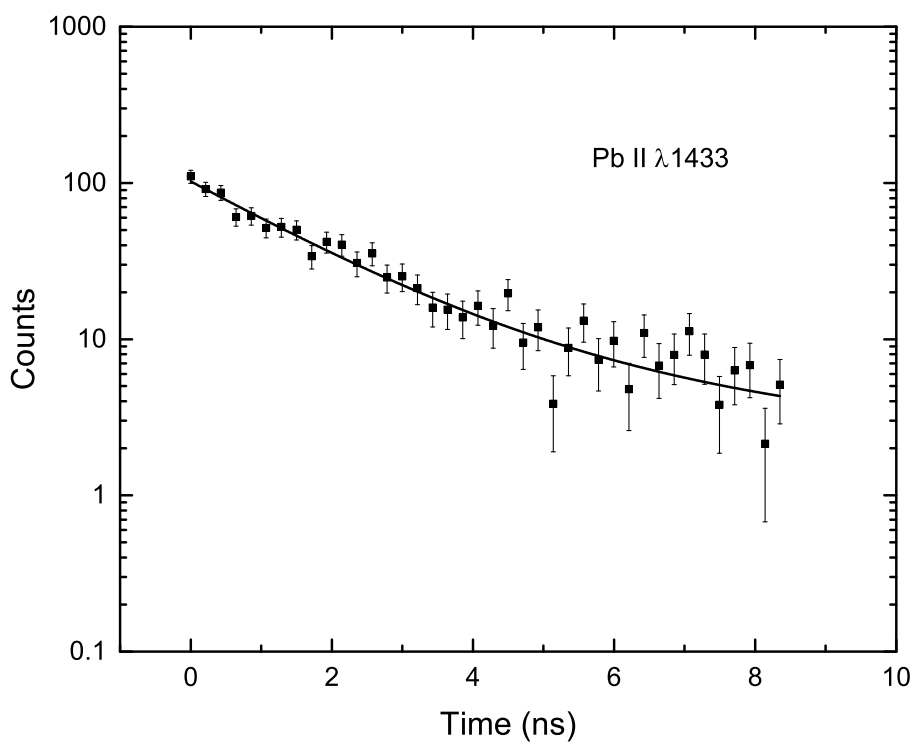

Fig. 3.- Decay curve for the $\lambda 1433$ line for a beam energy of $205 \mathrm{keV}$. The post-foil velocity ranges from $0.391 \mathrm{~mm} \mathrm{~ns}^{-1}$ to $0.392 \mathrm{~mm} \mathrm{~ns}^{-1}$ at this energy; these values were used to convert the foil position to the time. 


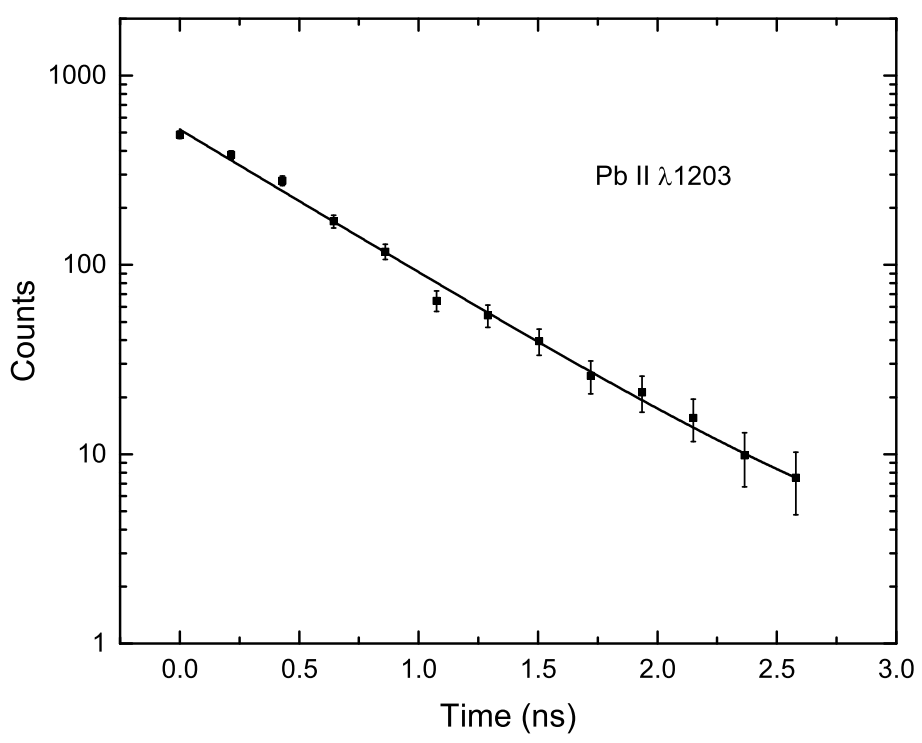

Fig. 4. - Same as Fig. 3 for the $\lambda 1203$ line for a beam energy of $205 \mathrm{keV}$ with a singleexponential fit as shown by the solid curve.

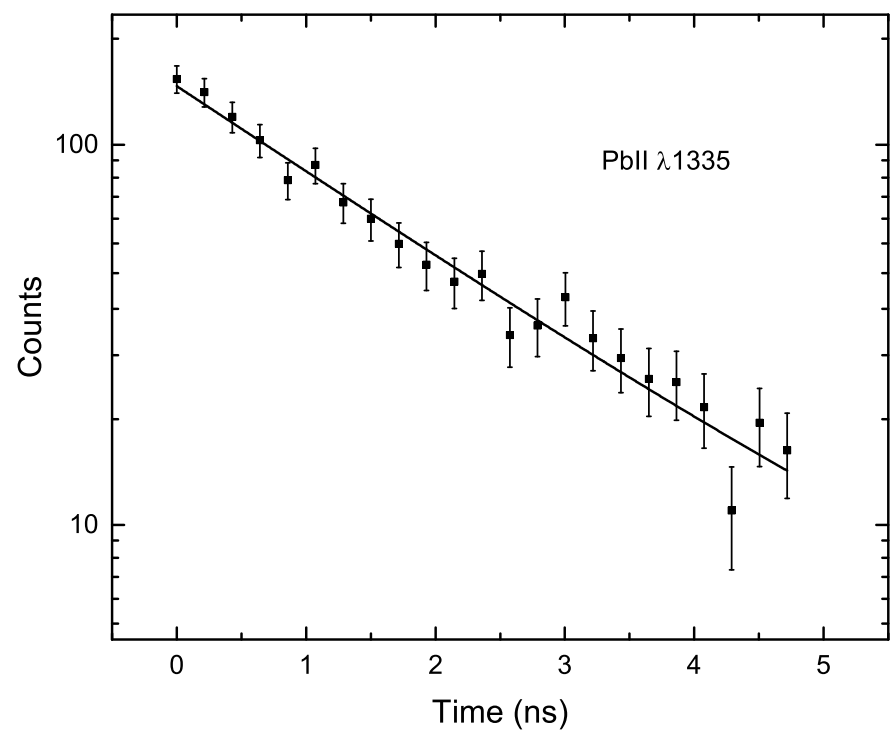

Fig. 5.- Same as Fig. 3 except for the $\lambda 1335$ line. 


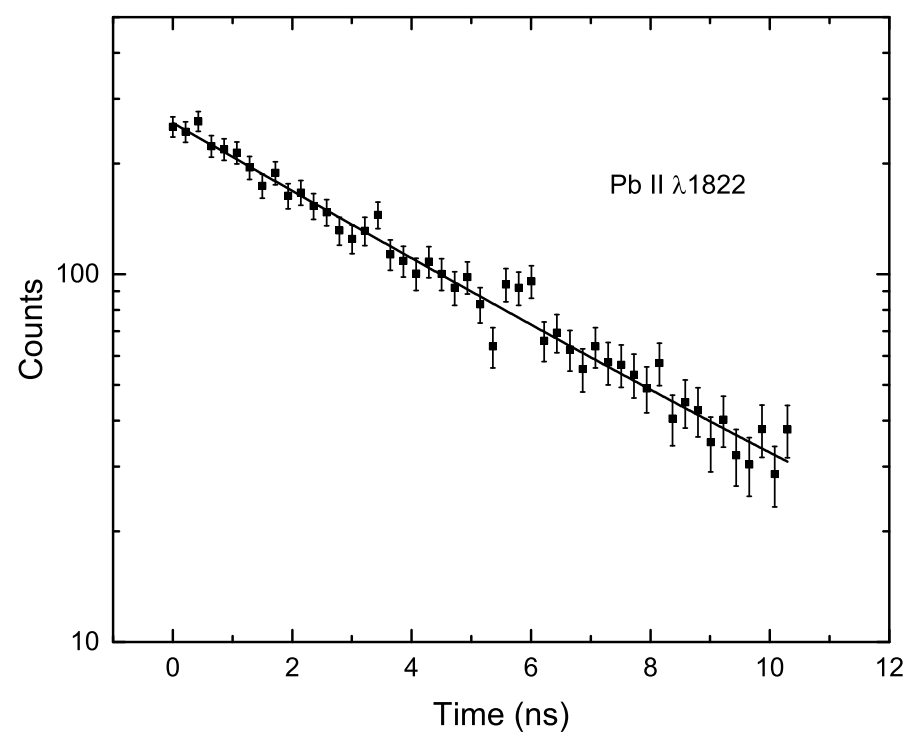

Fig. 6.- Same as Fig. 4 except for the $\lambda 1822$ line. 